\title{
A-STAR: THE ALL-SKY TRANSIENT ASTROPHYSICS REPORTER
}

\author{
J.P. Osborne ${ }^{1}$, P. O'Brien ${ }^{1}$, P. Evans ${ }^{1}$, G.W. Fraser ${ }^{1}$, A. Martindale ${ }^{1}$, \\ J.-L. Atteia ${ }^{2,3}$, B. Cordier ${ }^{4}$ and S. Mereghetti ${ }^{5}$
}

\begin{abstract}
The small mission A-STAR (All-Sky Transient Astrophysics Reporter) aims to locate the X-ray counterparts to ALIGO and other gravitational wave detector sources, to study the poorly-understood low luminosity gamma-ray bursts, and to find a wide variety of transient high-energy source types, A-STAR will survey the entire available sky twice per 24 hours. The payload consists of a coded mask instrument, Owl, operating in the novel low energy band $4-150 \mathrm{keV}$, and a sensitive wide-field focussing soft X-ray instrument, Lobster, working over $0.15-5 \mathrm{keV}$. A-STAR will trigger on $\sim 100 \mathrm{GRBs} / \mathrm{yr}$, rapidly distributing their locations.
\end{abstract}

\section{Introduction}

Responding to the 2012 call from the European Space Agency for a new type of small scientific mission for launch in late 2017, a consortium of institutes ${ }^{6}$ proposed A-STAR: the All-Sky Transient Astrophysics Reporter. The mission call had a cost cap of $€ 50 \mathrm{M}$ to ESA (including launch). Despite the severe cost limit, A-STAR is excitingly capable; it has three objectives:

1. Precisely locate the high-energy photon sources of gravitational-wave and neutrino transients and transients located by the new generation of astronomical facilities.

\footnotetext{
1 Dept of Physics \& Astronomy, University of Leicester, LE1 7RH, UK

2 UPS-OMP, Université de Toulouse, IRAP, Toulouse, France

3 CNRS, IRAP, 14 avenue Edouard Belin, 31400 Toulouse, France

4 CEA, IRFU, Service d'Astrophysique, 91191 Gif-sur-Yvette, France

5 INAF-IASF Milan, via Bassini 15, 20133 Milano, Italy

${ }^{6}$ UK: Univ. Leicester, ISIC; F: IRAP, LAM, ARTEMIS, CEA, IAP, APC; DK: DTU, SSC; I: IASF-Milan, IASF-Rome; B: Univ. Liège; CH: ISDC; PL: SRC (PAS).
} 
2. Reveal the physics underlying the variety in the population of gamma-ray bursts, including high-luminosity high-redshift bursts, low-luminosity bursts and short bursts.

3. Discover new high-energy transient sources over the whole sky, including supernova shock break-outs, black hole tidal disruption events, magnetar flares, and monitor known X-ray sources with daily observations.

\section{Scientific objectives}

\subsection{Gravitational wave sources}

The launch of A-STAR will coincide with the true dawn of the era of multimessenger astronomy during the second half of the current decade. The upgrading of the LIGO and VIRGO GW detectors (ALIGO/AVirgo) will revolutionize astronomy by permitting the detection and localization of GWs at a rate of perhaps dozens per year. IceCube and KM3NeT will likewise revolutionize neutrino astrophysics, routinely detecting cosmological neutrinos. Several of the most powerful sources of GWs predicted by general relativity, e.g. NS-NS or NS-BH mergers and core-collapse SNe, and neutrino sources such as GRBs produce powerful electromagnetic (EM) signals. ALIGO is planned to be operational by 2016-2018 and will be capable of identifying a randomly oriented NS-NS (NS-BH) merger out to $\sim 450(\sim 900) \mathrm{Mpc}$, with a combined predicted rate of $50 \mathrm{yr}^{-1}$ (Abadie et al. 2010), but with relatively poor sky localizations of $\sim 10-1000$ sq. degrees (Klimenko et al. 2011). IceCube and KM3NeT can localize to an accuracy of better than a few degrees but within a smaller volume of the Universe (the IceCube Collaboration 2011; Kappes 2007). To maximize the science return of the multi-messenger era requires an in-orbit trigger and search facility that can either simultaneously detect the event or rapidly observe the large error boxes provided by the GW and neutrino facilities with good sensitivity to the EM signal. This combined requirement is uniquely provided by A-STAR, which is able to trigger using $\mathrm{Owl}$ or Lobster and observe a very large fraction of the $\mathrm{GW} /$ neutrino error boxes within an orbit due to the large grasp of the Lobster instrument compared to current generation X-ray facilities, e.g. Lobster has a grasp 40 times that of Swift/XRT. For events triggered on-board with Owl or Lobster, GW searches can also be carried on the resultant known sky locations with lower ALIGO/AVirgo signal-to-noise thresholds and hence an increased search distance.

\subsection{Gamma-ray bursts}

In recent years GRBs have become essential in the study of stellar explosions, the evolution of massive stars (e.g. Pian et al. 2006), the extreme physics of relativistic jets and particle acceleration (e.g. Racusin et al. 2008), and as lighthouses illuminating the distant Universe (e.g. Tanvir et al. 2009). In the future we expect GRBs to provide a powerful probe of the epoch of reionization of the Universe (e.g. Tanvir et al. 2012), constrain the properties of the first stars, and will revolutionize GW astrophysics by associating GW signals with GRBs. 
In this context, it is striking that so little is known about the origin of GRBs and the conditions needed for their production. Many basic questions remain unresolved: Why do only a few percent of Type Ibc supernovae produce long GRBs? What is the role of metallicity and binarity in LGRB production? Are some LGRBs powered by proto-neutron stars, at least initially? What is the range of beaming angles of long and short GRBs? What progenitor systems produce short GRBs? Why do some nearby long GRBs have no accompanying supernova (Fynbo et al. 2006; Gal-Yam et al. 2006)? Having a functioning orbiting highenergy facility to address these issues is essential, particularly when we consider the advent of future large observatories such as the European ELT and the JWST at visible and infrared wavelengths, the full ALMA interferometer in the sub-mm, SKA and its precursors in radio, and CTA and HAWC at very high energies, that will revolutionize our vision of GRBs and their host galaxies. Other timedomain surveys such as Pan-STARRS and LSST will bring the power of multiwavelength observations to time domain astronomy, possibly revealing the elusive orphan afterglows of GRBs, permitting an accurate measure of GRB beaming.

\subsection{Discovering new high-energy transients}

Magnetars, young NS with external magnetic fields of $10^{13}-10^{15} \mathrm{G}$, are among the most powerful and spectacular high-energy transients in the sky. About twenty sources believed to be magnetars are currently known in our Galaxy and in the Magellanic Clouds, but since most of them are transients with long quiescent periods, the total population waiting to be discovered is certainly much larger (Mereghetti 2008). Magnetars can produce Giant Flares thought to be due to star crustal fractures, we predict that A-STAR will see more than 2 per year. Intermediate flares are more frequent; the wide field of view and the frequent sky coverage of ASTAR will, for the first time, allow detection of a large number of flares

Table 1. A-STAR detection rates for different astrophysical transients and variables.

\begin{tabular}{|l|l|}
\hline Transient type & Rate \\
\hline GW sources & $2-3 \mathrm{yr}^{-1}$ \\
\hline GRB & $100 \mathrm{yr}^{-1}$ \\
\hline Magnetars & $2 \mathrm{yr}^{-1}$ \\
\hline SN shock breakout & $1 \mathrm{yr}^{-1}$ \\
\hline TDE & $15 \mathrm{yr}^{-1}$ \\
\hline AGN+Blazars & 100 day $^{-1}$ \\
\hline Thermonuclear bursts & 10 day $^{-1}$ \\
\hline Novae & 1 \\
\hline Dwarf novae & day \\
\hline SFXTs & $10 \mathrm{yr}^{-1}$ \\
\hline Stellar flares & $\sim 100 \mathrm{yr}^{-1}$ \\
\hline Stellar super flares & $1 \mathrm{week}^{-1}$ \\
\hline
\end{tabular}
and obtain a reliable estimate of the frequency of such events.

The birth of a new SN is revealed by a burst of high-energy emission as the shock breaks out of the star. This has been spectacularly captured in a serendipitous Swift XRT observation of SN2008D (Soderberg et al. 2008): SNe are usually found only days to weeks after the explosion as radioactive heating powers optical brightening. Few observations exist early in a SN evolution: SN2008D remains 
the only non-GRB SN to be detected in X-rays at the time of first radiation escape from the star. A-STAR will significantly advance our understanding of the SN explosion mechanism, detecting SNe at the very moment of emergence, gathering comprehensive, prompt data and alerting follow-up communities to these landmark events.

Tidal disruption events (TDEs) offer a unique probe of the ubiquity of $\mathrm{BH}$ in galaxies, accretion on timescales open to direct study, and the nature and dynamics of galactic nuclei. Such events are expected to be visible as luminous, roughly Eddington limited objects with hot, UV and soft X-ray emission (e.g. Komossa et al. 2004; Gezari et al. 2012). The recent discovery by Swift of two highly luminous outbursts from galactic nuclei implies that at times a fraction of this energy is deposited in a new relativistic jet outflow (Levan et al. 2011; Bloom et al. 2011; Burrows et al. 2011), offering a new route to their identification and an opportunity to study newly-born jets. A-STAR is ideal for both the discovery and characterization of TDEs, opening new windows on numerous astrophysical questions.

\section{A-STAR instruments}

\subsection{Owl}

Owl is a coded mask telescope operating in the 4-150 keV energy range. It has a wide field of view $(\sim 1.44 \mathrm{sr})$ and a $10^{\prime}$ source error radius (90\% confidence) for the faintest sources ( $7 \sigma$ detection), improving to $2^{\prime}$ for the brightest $(>30 \sigma)$.

The Owl detector plane is made of 3840 Schottky CdTe detectors (4x $4 \mathrm{~mm} \times 1 \mathrm{~mm}$ thick) yielding a geometrical area of $614 \mathrm{~cm}^{2}$. The new generation ASICs developed at CEA Saclay, together with the careful detector selection and the optimized hybridization done at IRAP Toulouse allow to lower the detection threshold with respect to former CdTe detectors by about $10 \mathrm{keV}$, reaching $\sim 4 \mathrm{keV}$.

A coded aperture mask, made of a $0.6 \mathrm{~mm}$ thick tantalum sheet, placed

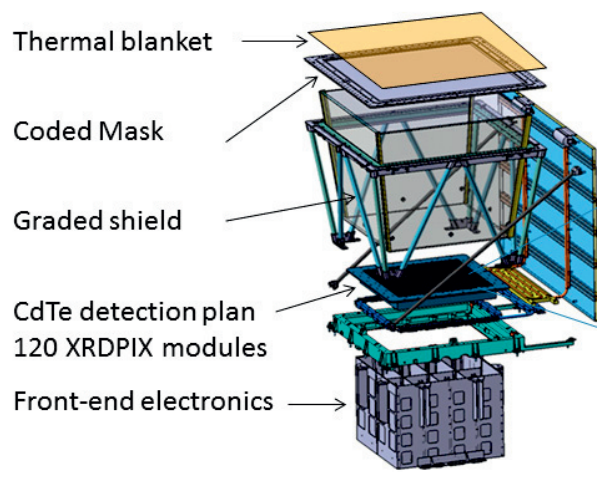

Fig. 1. The Owl codel mask instrument. $46 \mathrm{~cm}$ above this detection plane, defines a coded field of view of $\approx 60^{\circ} \times 88^{\circ}$. In order to optimize the sensitivity for short bursts the mask aperture was set at $40 \%$. Taking into account the geometrical parameters, and the materials present along the optical axis, the effective area is estimated to $104 \mathrm{~cm}^{2}$ at $4 \mathrm{keV}$.

Data are continuously analyzed on board in order to detect bursts, by first detecting a count rate increase (in several energy bands) on time scales from $10 \mathrm{~ms}$ to $20 \mathrm{~s}$ followed by the formation of the image in the triggered time window in 
which a new source is searched, or by systematic searches for new sources in images built on time scales from 20 to $1200 \mathrm{~s}$. Triggers are sent to the A-STAR service module to request a slew maneuver.

By decreasing its low energy threshold in the soft X-ray domain, while keeping a large field-of-view, $\mathrm{Owl}$ will open a new window on the Universe. It will detect almost all of the GRB population seen by Swift, and is especially sensitive to highly red-shifted bursts and to the poorly understood low energy bursts.

\subsection{Lobster}

Lobsters and other crustaceans focus by grazing incidence reflection off curved square pore optic arrays. This technique uniquely provides X-ray focussing over a very wide field, and is ideally suited to the A-STAR goals as it enables efficient detection of a large number of GRBs in a new low energy regime.

Lobster comprises 3 modules, each with a $17.3 \times$ $17.3^{\circ}$ field of view. These form a single 900 square degree FOV, centred on that of Owl. Each Lobster module has an array of $7 \times 740 \times 40 \mathrm{~mm}$ Micro-Channel Plate optics, mounted onto a titanium front end, supported by a carbon fibre mechanical structure. The optics have a spherical radius of $600 \mathrm{~mm}$, focusing onto the detector at $300 \mathrm{~mm}$. The camera contains an MCP detector which is curved to match the $300 \mathrm{~mm}$ curvature of the focal plane, an anti-coincidence system, a thin aluminized

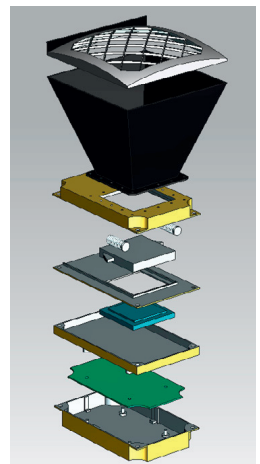

Fig. 2. A single Lobster module. polyimide optical/UV blocking filter, and the readout and analogue electronics. This configuration has a position resolution FWHM of $25 \mu \mathrm{m}$ over the $\sim 93 \times 93 \mathrm{~mm}$ imaging area of the detector, sufficient to significantly oversample the PSF.

Lobster provides un-vignetted and uniform resolution imaging across a very wide FOV, while maintaining the imaging advantage in sensitivity over collimating and coded mask systems. The point spread function of a Lobster optic is cruciform, $25 \%$ of the counts fall in the central peak, $50 \%$ in the arms and $25 \%$ in a diffuse centred pyramid. The detector performance is well matched to the optic capabilities and allows source position centroiding to $<1.8^{\prime}$ for $90 \%$ of GRBs, $<0.5^{\prime}$ for $50 \%$ and $10 \%$ better than $<0.17^{\prime}$ for $10 \%$ (determined by Monte-Carlo simulation of Lobster observation of Swift GRBs).

The position and trigger algorithm maximises sensitivity by employing two stages: the first projects the image in two perpendicular 1D histograms, and a candidate detection is when a $2.5 \sigma$ event is seen above background in both axes. The second stage takes a cross shaped patch centred on the candidate and integrates over time, the transient is confirmed if the signal exceeds a specifiable higher statistical significance (e.g. $\sim 6.3 \sigma$ to achieve a false trigger rate of 1 in $\left.10^{10}\right)$. At this level the 30 -minute sensitivity is $3 \times 10^{-11}$ erg. $\mathrm{cm}^{-2}$.s $(0.15-5 \mathrm{keV})$. The twostage approach maximises sensitivity to faint transients and minimises false alerts. 


\section{Mission profile}

A-STAR carries two wide-field X-ray imagers and a fast communication system. The payload is designed to operate with the mass and power resources provided by a microsatellite platform. We have performed detailed accommodation studies, which have demonstrated that the Myriade Evolutions and Proba satellites fit within a standard Vega piggyback volume. The operational mission life is 3 years.

The scientific return of A-STAR depends crucially upon the ability of the satellite to point the instruments at the open sky for 15-30 min long exposures; the ability to compute the positions of detected transients on-board and to transmit alerts quickly to the ground; and a fast reaction on reception of Targets of Opportunity. Key to success is the number of transients detected; the mission profile is optimized to maximize this number. We have therefore chosen an orbit with an altitude of $650 \mathrm{~km}$ and an inclination $\mathrm{i}<30^{\circ}$ in order to minimize the time spent un-

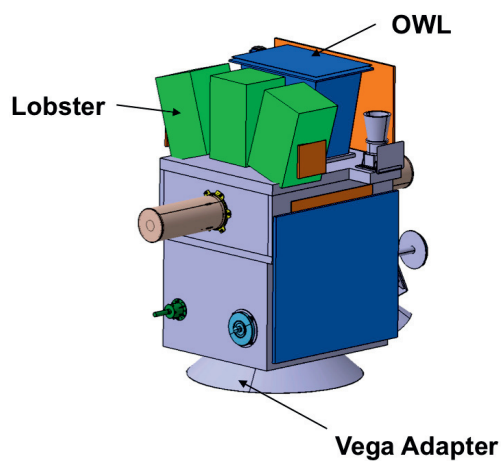

Fig. 3. A-STAR on Myriade Evolution. usably in a high radiation environment. This orbit can be reached with a Vega launch as a passenger (A-STAR fits within the standard piggyback volume).

The survey strategy is an essential ingredient of the mission. A-STAR will cover a significant fraction of the sky twice a day, perform long exposures taking full advantage of Lobster sensitivity, and observe Owl-detected transients with Lobster within 1-2 minutes. The A-STAR strategy relies on three $\sim 1500 \mathrm{sec}$ long dwells per orbit, two in the day side and one in eclipse, respecting the $90^{\circ}$ sun avoidance angle required by $\mathrm{Owl}$ and Lobster. The eclipse dwells will detect transients in the night hemisphere, allowing a prompt response by ground-based facilities.

The need to transmit alerts quickly (within 1 minute) from the spacecraft to the ground calls for a dedicated system. We have studied two possible systems: in the first option, the consortium provides a network of VHF ground stations, as studied by CNES for the SVOM mission. An alternative is to use the COM Dev (Europe) SB-Sat system to send short messages via Inmarsat.

\section{Concluding remarks}

ESA announced that A-STAR was not selected on October 15, 2012. We believe that the science case for a wide-field high-cadence X-ray survey is very strong, and in particular that the promise of the new generation of gravitational wave detectors is best realised by such a survey. We intend to pursue future new mission opportunities vigorously. 
We thank the 29 Co-Is and 62 associate scientists for their valuable input to the A-STAR proposal. JPO \& PE acknowledge the support of the UK Space Agency.

\section{References}

Abadien, J., Abbot, B.P., Abbot, R., et al., 2010, Class. Quantum Grav., 27, 173001

Klimenkon, S., Vedovato, G., Drago, M., et al., 2011, PhRvD, 83, 102001

The IceCube Collaboration, 2011 [arXiv:1111.2741]

Kappes, A., 2007 [arXiv:0711.0563]

Pian, E., Mazalli, P.A., Masetti, N., et al., 2006, Nature, 442, 1011

Racusin, J.L., Karpov, S.V., Sokolowski, M., et al., 2008, Nature, 455, 183

Tanvir, N.R., Fox, D.B., Levan, A.J., et al., 2009, Nature, 461, 1254

Tanvir, N.R., Levan, A.J., Fructer, A.S., et al., 2012, ApJ, 754, 46

Fynbo, J.P.U., Watson, D., Thöne, C.C, et al., 2006, Nature, 444, 1047

Gal-Yam, A., Fox, D.B., Price, P.A., et al., 2006, Nature, 444, 1053

Mereghetti, S., 2008, A\&ARv, 15, 225

Soderberg, A.M., Berger, E., Page, K.L., et al., 2008, Nature, 453, 469

Komossa, S., Halpern, J., Schartel, N., et al., 2004, ApJ, 603, L17

Gezari, S., Chornock, R., Rest, A., et al., 2012, Nature, 485, 217

Levan, A.J., Tanvir, N.R., Cenko, S.B., et al., 2011, Science, 333, 199

Bloom, J.S., Giannios, D., Metzger, B., et al., 2011, Science, 333, 203

Burrows, D.N., Kennea, J.K., Ghisellini, G., et al., 2011, Nature, 476, 421 
